\title{
Ume (Japanese Apricot)- Induced Small Bowel Obstruction with Chronic Radiation Enteritis
}

\author{
Takuya Hashimoto $^{\text {a Joji Kitayama }}{ }^{\text {a }}$ Akio Hidemura $^{a}$ \\ Hironori Ishigami $^{a}$ Shoichi Kaizaki ${ }^{a}$ Noriyoshi Fukushima ${ }^{b}$ \\ Tetsuro Miyata $^{\mathrm{a}}$ Hirokazu Nagawa ${ }^{\mathrm{a}}$ \\ Departments of aSurgery and 'bPathology, Graduate School of Medicine, \\ The University of Tokyo, Tokyo, Japan
}

\section{Key Words}

Radiation injury · Intestinal obstruction · Foreign bodies · Enteritis

\begin{abstract}
Stricture formation is recognized as one of the complications of chronic radiation enteritis. Here, we present a case of a 73-year-old woman who presented with small bowel obstruction 16 years after pelvic irradiation for uterine cancer. Computed tomographic (CT) scan of the abdomen demonstrated a $1-\mathrm{cm}$ foreign body in the terminal ileum. Laparotomy revealed a stone of ume (Japanese apricot) stuck in an ileal stricture, leading to complete impaction and perforation. She was successfully treated with ileocecal resection and ileocolic anastomosis without any complication.

Pathological study revealed that the low compliance caused by fibrosis of the bowel wall prevented the small ume stone from passing through the irradiated ileum. Our case implies the specific risk of food-induced small bowel obstruction in patients with a history of pelvic irradiation.
\end{abstract}

\section{Introduction}

Currently, $60 \%$ of small bowel obstructions are accounted for by adhesions with a great increase in elective abdominal surgery from the late 20th century. The remaining causes include hernias $15 \%$, neoplasms $6 \%$, inflammatory causes $5 \%$, mesenteric vascular occlusions 5\%, intussusception 3\%, and unusual etiology such as foreign bodies $6 \%$ [1]. 
On the other hand, since first described in 1897, radiation is well known to have toxic gastrointestinal effects [2]. Among them, stricture formation is recognized as one of the complications of chronic radiation enteritis. It is easily conceivable that intestinal narrowing can be a contributing factor to the obstruction by foreign bodies.

Here, we present a case of a 73-year-old woman who presented with small bowel obstruction 23 years after pelvic irradiation for uterine cancer. It was the stricture caused by fibrosis of the bowel wall that prevented the accidentally swallowed small ume stone from passing through the irradiated ileum.

\section{Case Report}

A 73-year-old woman, who had undergone total hysterectomy and pelvic radiation therapy with a total dose of $50 \mathrm{~Gy}$ for uterine cancer 23 years earlier, was hospitalized for nausea and abdominal fullness. She had other past history including iliac artery occlusion presumably due to radiation and rheumatoid arthritis which had been treated with $5 \mathrm{mg}$ of predonisolone per day for 23 years.

On examination her abdomen was distended without guarding or rebound tenderness. Bowel sounds were increased and tinkling. The hernial orifices were normal. Temperature was $36.9^{\circ} \mathrm{C}$, pulse 66 beats/min, and blood pressure 120/58 $\mathrm{mm} \mathrm{Hg}$. Laboratory studies revealed a high white blood cell count of $11,500 / \mathrm{mm}^{3}$ and a low albumin level of $2.5 \mathrm{mg} / \mathrm{dl}$.

$\mathrm{X}$-ray showed small bowel dilatation, suggesting mechanical obstruction. CT scan of the abdomen and pelvis showed a nut shell-like high-density structure in the small bowel (fig. 1). The patient said that she had accidentally swallowed a kernel of ume (Japanese apricot) a few days prior to the onset of the symptom. She was subjected to surgery with a diagnosis of food-induced intestinal obstruction.

At laparotomy, we found impaction of a solid stone $(13 \times 18 \mathrm{~mm})$ causing perforation of the sclerosed ileum with a whitish appearance, $50 \mathrm{~cm}$ proximal to the ileocecal valve (fig. $2 \mathrm{a}$ ). Ileocecal resection was performed and she made an uneventful recovery. The resected ileum showed severe luminal stenosis and had two mucosal ulcerations corresponding to the protrusions of the ume seed, one of which resulted in a pin-hole perforation (fig. 2b). Histopathologic examination of the resected ileum with stricture $(10 \mathrm{~cm}$ distal to the perforation area) showed fibrosis of the lamina propria (fig. $3 \mathrm{a}$, arrow) and moderate submucosal edema. Villous shortening and flattening were also prominent. These histopathological features were consistent with the diagnosis of chronic radiation enteritis. Figure $3 \mathrm{~b}$ shows severe inflammation and mucosal ulceration of the perforation area $50 \mathrm{~cm}$ proximal to the ileocecal valve. It is considered that radiation-induced fibrosis reduced the compliance of the ileal wall, which prevented passage of the small ume stone.

\section{Discussion}

Radiation enteritis generally affects the distal ileum, sigmoid colon and rectum [3] because the pelvic region is the most frequent target of radiotherapy for tumors such as gynecologic cancer. In the acute phase, radiation mainly affects the intestinal epithelium [4] and induces edema, hyperemia and ulceration, leading to severe diarrhea. In contrast, chronic radiation damage is caused by progressive endarteritis obliterans involving the submucosal and serosal layers [5], followed by segmental ischemia of the bowel wall and replacement by hyaline fibrous tissue that often results in obstructive symptoms.

The incidence of chronic radiation enteritis depends on the size of the irradiated field and the total dose [6]. It has been reported that clinical manifestations of chronic injury to the intestine occurred in 20 to $30 \%$ of cases treated with 50 to $60 \mathrm{~Gy}$ and one third or more of those treated with more than $60 \mathrm{~Gy}$ [7]. Our patient received $50 \mathrm{~Gy}$ to the whole pelvis, a typical dose for uterine carcinoma. The latent period of these manifestations may range from 6 months to 25 years [8]. Our patient experienced no gastrointestinal 
symptoms for 23 years after irradiation, although she developed iliac artery stenosis possibly caused by the same mechanisms.

In our case, the rigid wall and stricture formation as well as histological findings in the terminal ileum were compatible with chronic changes after irradiation. The accidentally swallowed ume stone became stuck in the stricture in the ileum, causing ulceration and finally perforation. A literature search revealed numerous reports about phytobezoars, which are the common cause of food-induced obstruction, but none made mention of an association with radiation enteritis. However, there are 5 case reports of food-induced small bowel obstruction with radiation enteritis in the Japanese literature. Interestingly, all 5 cases were caused by a single ume stone. Pickled ume, a traditional Japanese food often served with rice as a relish, is especially eaten by the Japanese elderly. The size of the seeds ranges from approximately 0.8 to $2 \mathrm{~cm}$ in diameter, which usually pass through the whole bowel even when swallowed. However, our case suggests that the low compliance caused by pathological changes did not allow the ingested ume stone to pass through the irradiated ileum.

Complications of chronic radiation enteritis present a significant clinical problem because of technical difficulties in surgical treatment. Therefore, in cases with obstruction, conservative treatment consisting of intravenous fluids and nasogastric tube decompression is generally recommended at first. However, resection of ischemic and fibrotic bowel is necessary for patients like our case with food impaction. It is controversial whether bowel reconstruction should be performed at the same time or whether judicious stoma formation should be performed to avoid anastomosis leakage, since wound healing is considered to be impaired in irradiated bowel [9]. Galland et al. suggested the advantage of treatment of terminal ileal lesions by right hemicolectomy and ileo-transverse anastomosis [10]. In fact, in patients with pelvic radiotherapy, the ascending, transverse and descending colon are usually free from radiation and are preferable sites for anastomosis. Nowadays, resection with primary anastomosis is generally favored if anastomosis can be performed in uninvolved intestine. In our case, we selected ileo-cecal resection, and anastomotic leakage did not occur despite malnutrition and the regular use of steroids.

In conclusion, when elderly with a past history of pelvic radiation therapy present with small bowel obstruction, the possible influence of late radiation injury to the intestine should be considered. The patient should be carefully interviewed about ingested material because even small objects can become stuck in a stricture in the ileum. When surgical management is required, anastomosis should be performed in bowel free of radiation injury. 
Fig. 1. Enhanced CT scan of abdomen and pelvis demonstrated a high density structure shaped like a nut shell in the small bowel (white arrow).

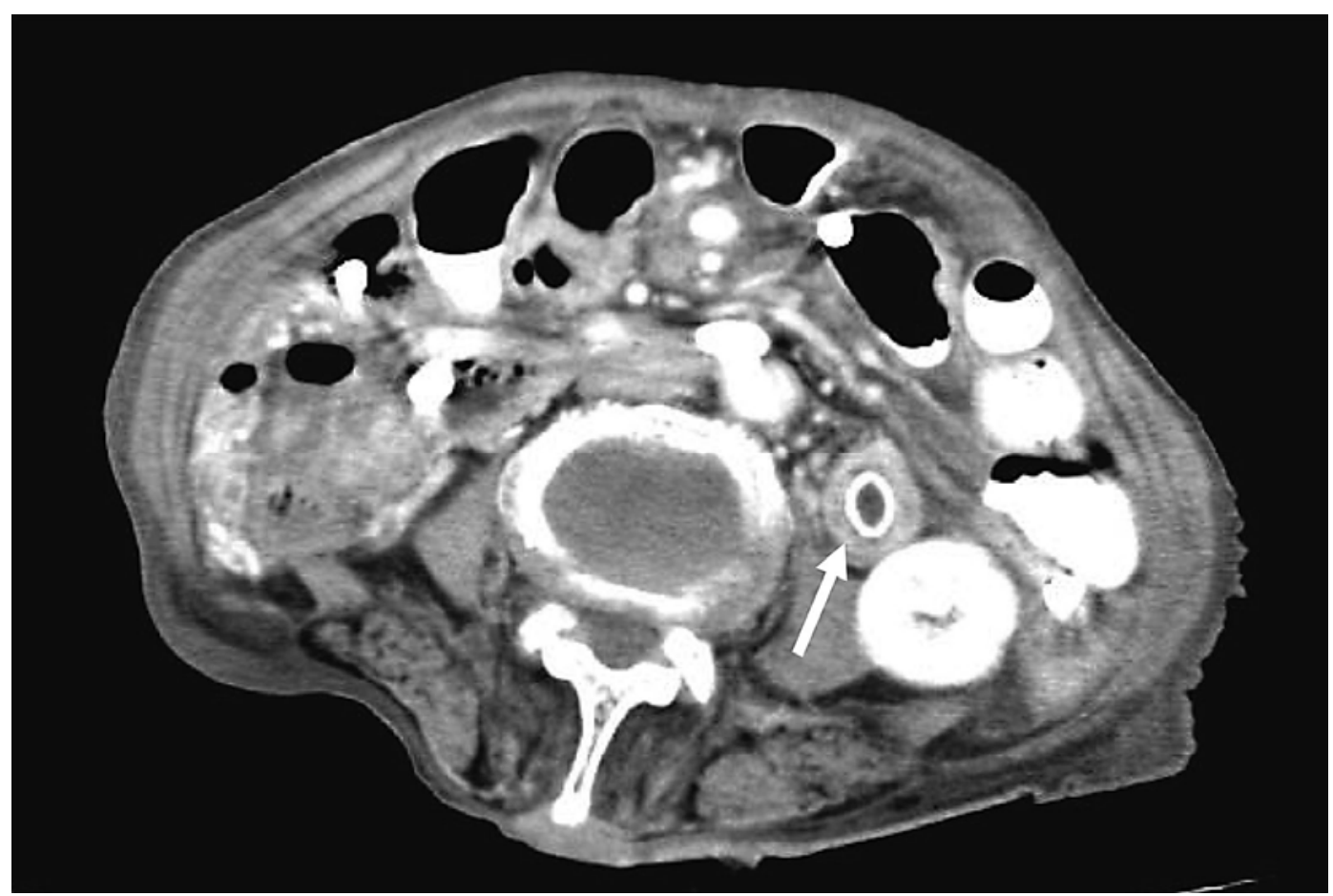

Fig. 2. a Perforated ileum with a whitish appearance (white arrow). b The resected ileum had two mucosal ulcerations corresponding to the protrusions of the ume seed, one of which resulted in a pinhole perforation (arrow).
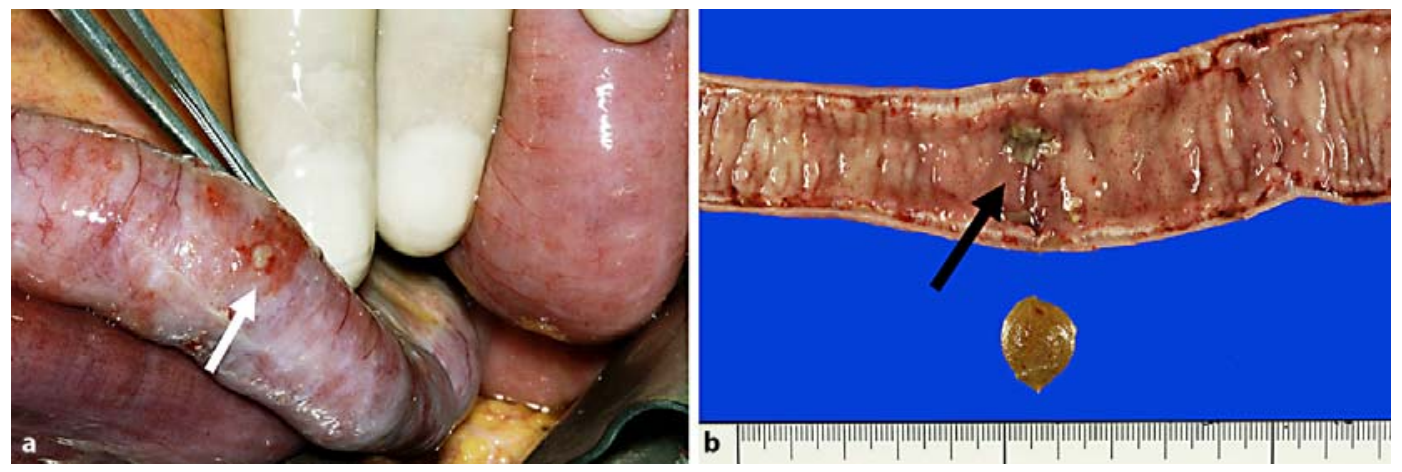


\begin{tabular}{r|l|l|l}
$\begin{array}{r}\text { Case Reports in } \\
\text { Gastroenterology }\end{array}$ & $\begin{array}{l}\text { Case Rep Gastroenterol 2007;1:184-189 } \\
\text { D0I: 10.1159/000112653 }\end{array}$ & Published online: December 31, 2007 & $\begin{array}{l}\text { O 2007 S. Karger AG, Basel } \\
\text { ISSN 1662-0631 } \\
\text { www.karger.com/crg }\end{array}$ \\
\hline
\end{tabular}

Fig. 3. Histologic findings of resected intestinal wall (hematoxylin and eosin, original magnification $\times 40$, bar indicates $0.2 \mathrm{~mm}$ ). a Fibrosis of lamina propria in the terminal ileum (arrows). Villous shortening is compatible with chronic radiation damage. b Severe inflammation and mucosal ulceration caused by a seed.

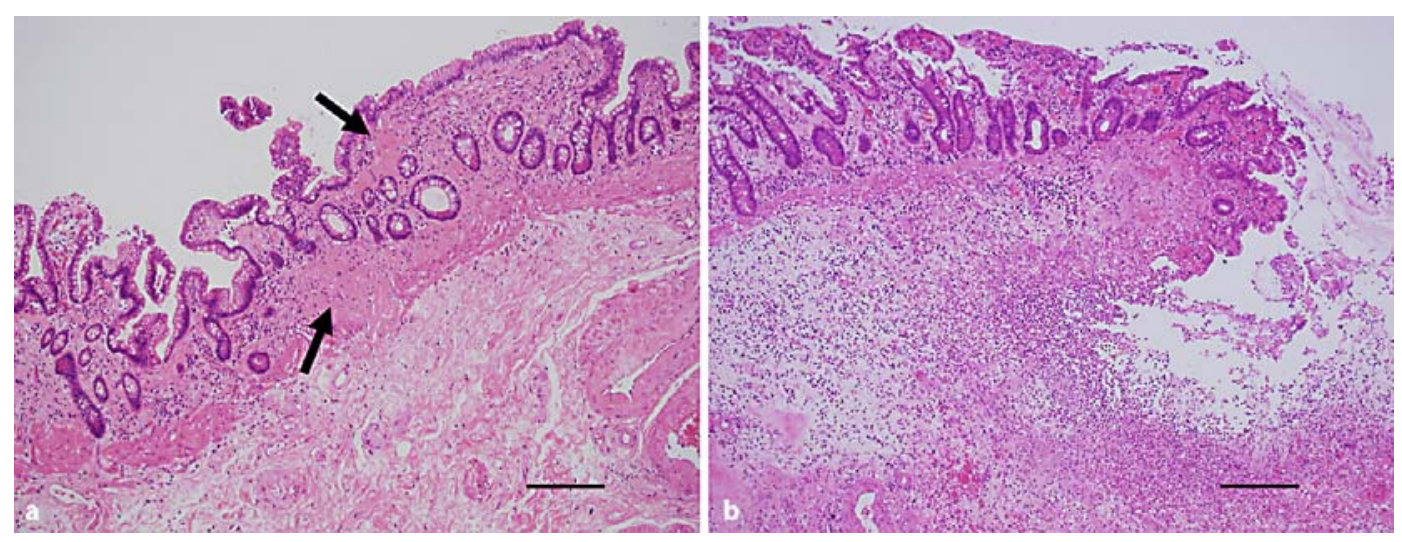




\section{References}

1 Lohn JW, Austin RC, Winslet MC: Unusual causes of small-bowel obstruction. J R Soc Med 2000;93:365-368.

2 Walsh D: Deep tissue traumatism from roentgen ray exposure. Br Med J 1897;2:272-273.

- 3 Yoshimura K, Hirata I, Maemura K, Sugi K, Tahara T: Radiation enteritis: a rare complication of the transverse colon in uterine cancer. Intern Med 2000;39:10601063.

4 MacNaughton WK: Review article: new insights into the pathogenesis of radiation-induced intestinal dysfunction. Aliment Pharmacol Ther 2000;14:523528.

5 Novak JM: Effects of radiation on the human gastrointestinal tract. J Clin Gastroenterol 1979;1:9-39.

-6 Classen J: Radiation-induced gastrointestinal toxicity. Pathophysiology, approaches to treatment and prophylaxis. Strahlenther Onkol 1998;174(suppl 3):82-84.

7 Berthrong M, Fajardo LF: Radiation injury in surgical pathology. Part II. Alimentary tract. Am J Surg Pathol 1981;5:153-178.

8 Nguyen NP, Antoine JE, Dutta S, Karlsson U, Sallah S: Current concepts in radiation enteritis and implications for future clinical trials. Cancer 2002;95:11511163.

9 Cross MJ, Frazee RC: Surgical treatment of radiation enteritis. Am Surg 1992;58:132-135.

10 Galland RB, Spencer J: Natural history and surgical management of radiation enteritis. Br J Surg 1987;74:742-747. 